\title{
ELECTRONIC DAMPING OF MICROPHONICS IN SUPERCONDUCTING CAVITIES*
}

\author{
J. R. Delayen ${ }^{\dagger}$, Thomas Jefferson National Accelerator Facility, Newport News, VA 23606, USA
}

\section{Abstract}

In previous applications of high-velocity superconducting cavities, the accelerated beam currents were sufficiently high that the microphonics-induced frequency excursions were significantly less than the loaded bandwidth, and the power absorbed by the beam dominated the total power requirement. In new applications (CEBAF Upgrade, RIA) the beam currents will be sufficiently low that the rf power requirements will be dominated by the control of the cavity fields in the presence of microphonics. Active electronic damping of microphonics by modulation of the cavity field amplitude has been occasionally used in the past in small, lowvelocity, low-gradient superconducting structures; its application to much larger, high-velocity, high-gradient structures could result in a substantial reduction of the $\mathrm{rf}$ power requirements. This paper presents an analytical study of various schemes for electronic damping and presents formulae to quantify the reduction of microphonics as a function of rf field modulation.

\section{INTRODUCTION}

One of the early challenges in the application of $\mathrm{rf}$ superconductivity to particle accelerators, especially ion accelerators, was the control and stabilization of the phase and amplitude of the accelerating fields in the large number of independent cavities. Ambient noise and microphonics can cause frequency variations that are larger than the bandwidth of the resonators.

Historically, high-velocity superconducting resonators have been used in high-current applications where most of the rf power was transferred to the beam. Recently, however, high-velocity elliptical-type resonators will be used in applications where the beam loading will be small and the need for phase and amplitude control will dictate most or all the requirements for $\mathrm{rf}$ power.

In the case of no beam loading, the minimum amount of rf power required for phase stabilization by negative feedback is given by $P=U \delta \omega$ [1-3], where $U$ is the energy content at operating gradient, and $\delta \omega$ is the maximum amount of detuning at which phase lock is to be maintained. $\delta \omega$ has two components: a static component given by the accuracy with which the average cavity resonance frequency can be matched to the master reference frequency, and a dynamic component due to microphonics-induced frequency excursions. With a welldesigned mechanical tuning system, the static component

* This work was supported by the U.S. DOE Contract No DE-AC0584-ER40150.

†delayen@jlab.org can be made much smaller than the dynamic component, and any reduction of the microphonics would lead to a corresponding reduction in the rf power requirements.

Mechanical stiffening of resonators is often used to reduce the Lorentz detuning and may also reduce, to some extent, the microphonics. Mechanical damping has been very effective in some low-velocity structures [4], but has not yet been implemented in elliptical-type cavities. Electronic damping has also been used in low-velocity structures [2]; in this paper we present an analytical study of electronic damping as a possible means of reducing microphonics in superconducting cavities.

\section{MODEL AND EQUATIONS}

We will use the same model described in more details in [1-3], namely a resonator operated in a self-excited loop. In [1] it was found advantageous to operate the loop slightly off resonance on the low frequency side $\left(\theta_{l}<0\right)$; this introduced a small amount of coupling between phase and amplitude feedback which could be used to damp the microphonics. In [3] a feedback phase shifter $\left(\theta_{f}\right)$ was added that could be used to provide the same amount of coupling while still operating the unlocked self-excited loop on resonance $\left(\theta_{l}=0\right)$.

A block diagram of such a configuration is shown in Figure 1, and a transfer function representation of the system is shown in Figure 2 where [3]:

$$
G_{a a}=\frac{\cos \left(\theta_{l}+\theta_{f}\right)}{\cos \theta_{l}} \frac{1}{1+\tau s}, \quad G_{t a}=-\frac{\sin \left(\theta_{l}+\theta_{f}\right)}{\cos \theta_{l}} \frac{1}{1+\tau s},
$$$$
G_{a \omega}=\frac{1}{\tau} \frac{\cos \left(\theta_{l}+\theta_{f}\right)}{\cos \theta_{l}}\left[y-\frac{y_{r}}{1+\tau s}\right], G_{t \omega}=\frac{1}{\tau} \frac{\cos \left(\theta_{l}+\theta_{f}\right)}{\cos \theta_{l}}\left[1+\frac{y y_{r}}{1+\tau s}\right],
$$$$
G_{\mu}=-\frac{2 \Omega_{\mu}^{2} k_{\mu} V_{0}^{2}}{s^{2}+\frac{2}{\tau_{\mu}} s+\Omega_{\mu}^{2}} \quad, \quad G_{b a}=-\frac{m}{1+\tau s} \quad, \quad G_{\varphi a}=\frac{m y_{0}}{1+\tau s},
$$

$G_{b \omega}=\frac{m}{\tau}\left[-y_{0}+\frac{y_{r}}{1+\tau s}\right], \quad G_{\varphi \omega}=-\frac{m}{\tau}\left[1+\frac{y_{0} y_{r}}{1+\tau s}\right]$,

$F_{a}$ : Amplitude Feedback, $\quad F_{\varphi}$ : Phase Feedback ,

$\tau=\frac{\tau_{0}}{1+\beta}:$ Loaded amplitude decay time,

$b=\frac{V_{b} \cos \varphi_{0}}{V_{0}}$ : ratio of power absorbed by the beam to

power dissipated in the cavity,

$m=\frac{b}{1+\beta} \quad$ : Beam matching coefficient.

$y_{0}=\tan \theta_{0}, y_{l}=\tan \theta_{l}, y=\tan \left(\theta_{l}+\theta_{f}\right), y_{r}=\frac{\tau_{0}\left(\omega_{r}-\omega_{c}\right)}{1+\beta}$ 
$G_{\mu}$ represents the coupling between the field amplitude and cavity frequency which is responsible for the ponderomotive instabilities [1]. $\Omega_{\mu}$ is the frequency of the mechanical mode of the cavity, and $\tau_{\mu}$ is its decay time.

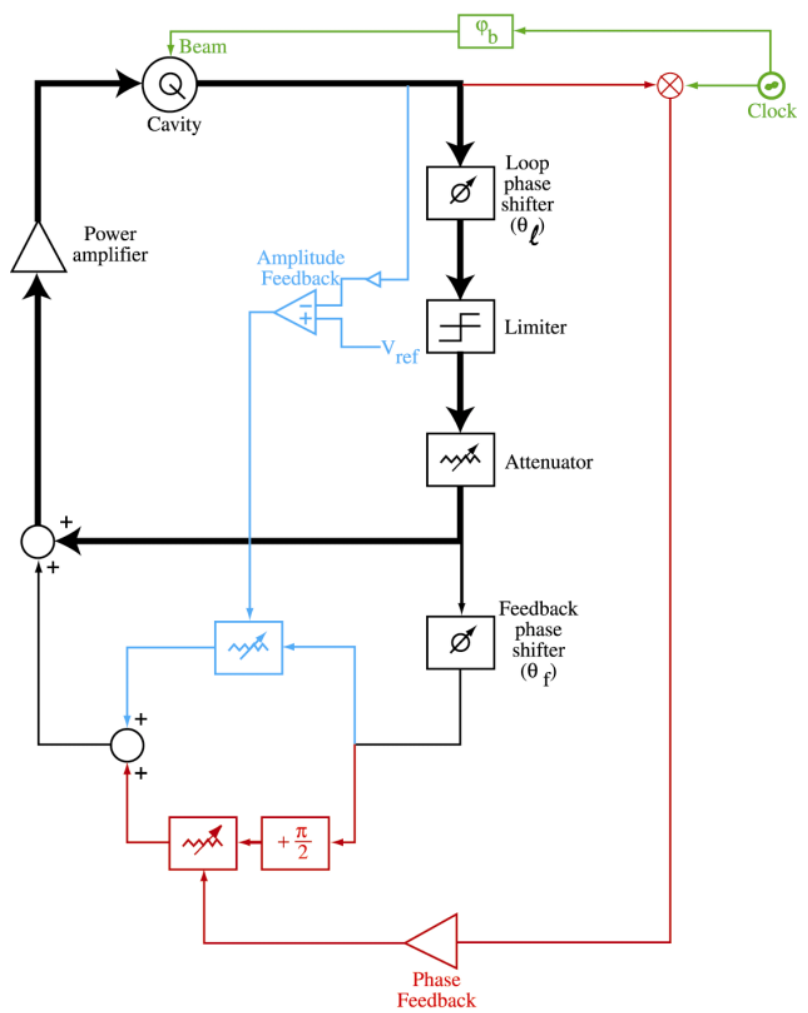

Figure 1: Block diagram of a resonator operating in a selfexcited loop in the presence of beam loading with phase and amplitude feedback

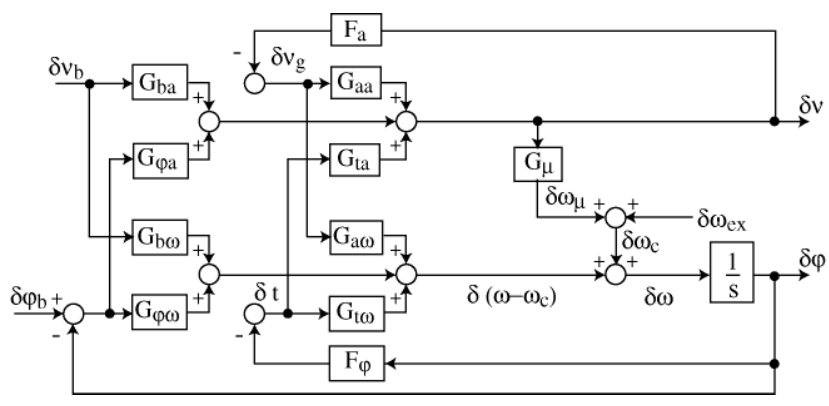

Figure 2: Transfer function representation of the system shown in Figure 1.

The residual amplitude and phase errors due to fluctuations of the cavity eigenfrequency $\left(\delta \omega_{e x}\right)$, beam current $\left(\delta v_{b}\right)$ and beam phase $\left(\delta \varphi_{b}\right)$ are

$$
\begin{aligned}
\delta v=D^{-1}\{ & -\delta \omega_{e x}\left(G_{\varphi a}+F_{\varphi} G_{t a}\right) \\
& +\delta_{v_{b}}\left[G_{b a}\left(s+G_{\varphi \omega}+F_{\varphi} G_{t \omega}\right)-G_{b \omega}\left(G_{\varphi a}+F_{\varphi} G_{t a}\right)\right] \\
& \left.+\delta \varphi_{b}\left[G_{\varphi a}\left(s+G_{\varphi \omega}+F_{\varphi} G_{t \omega}\right)-G_{\varphi \omega}\left(G_{\varphi a}+F_{\varphi} G_{t a}\right)\right]\right\} \\
\delta \varphi=D^{-1}\{ & \delta \omega_{e x}\left(1+F_{a} G_{a a}\right) \\
& +\delta_{v_{b}}\left[G_{b \omega}\left(1+F_{a} G_{a a}\right)-G_{b a}\left(F_{a} G_{a \omega}-G_{\mu}\right)\right] \\
& \left.+\delta \varphi_{b}\left[G_{\varphi \omega}\left(1+F_{a} G_{a a}\right)-G_{\varphi a}\left(F_{a} G_{a \omega}-G_{\mu}\right)\right]\right\}
\end{aligned}
$$

with

$D=\left(1+F_{a} G_{a a}\right)\left(s+G_{\varphi \omega}+F_{\varphi} G_{t \omega}\right)-\left(G_{\varphi a}+F_{\varphi} G_{t a}\right)\left(F_{a} G_{a \omega}-G_{\mu}\right)$.

\section{PERFORMANCE OF STABILIZATION SYSTEM}

If we assume that the fluctuations in resonator field phase and amplitude are due to fluctuations in cavity eigenfrequency, and that these in turn are due to the excitation of the mechanical mode by white noise of spectral density $A^{2}$, then the mean square values for the cavity frequency, and field amplitude and phase are given by [1]:

$$
\begin{aligned}
& <\delta \omega_{e x}^{2}>=A^{2} \int_{-\infty}^{+\infty}\left|-\omega^{2}+\frac{2}{\tau_{\mu}} i \omega+\Omega_{\mu}^{2}\right|^{-2} d \omega=A^{2} \frac{\pi \tau_{\mu}}{2 \Omega_{\mu}^{2}} \\
& <\delta v^{2}>=<\delta \omega_{e x}^{2}>\frac{2 \Omega_{\mu}^{2}}{\pi \tau_{\mu}} \int_{-\infty}^{+\infty}\left|\frac{G_{a}(i \omega)}{-\omega^{2}+\frac{2}{\tau_{\mu}} i \omega+\Omega_{\mu}^{2}}\right|^{2} d \omega \\
& <\delta \varphi^{2}>=<\delta \omega_{e x}^{2}>\frac{2 \Omega_{\mu}^{2}}{\pi \tau_{\mu}} \int_{-\infty}^{+\infty}\left|\frac{G_{\varphi}(i \omega)}{-\omega^{2}+\frac{2}{\tau_{\mu}} i \omega+\Omega_{\mu}^{2}}\right|^{2} d \omega
\end{aligned}
$$

where $G_{a}=-\left(G_{\varphi a}+F_{\varphi} G_{t a}\right) D^{-1}$ and

$G_{\varphi}=\left(1+F_{a} G_{a a}\right) D^{-1}$.

The mean square errors $\left\langle\delta v^{2}\right\rangle$ and $\left\langle\delta \varphi^{2}\right\rangle$ can be calculated in the most general case but we will present the results under the following assumptions: no beam loading, loop phase adjusted so the unlocked cavity operates on resonance $\left(\theta_{l}=0\right)$, small feedback angle $\left(\theta_{f} \ll 1\right)$, large proportional feedback gains $\left(k_{a}, k_{\varphi} \gg 1, \tau \Omega_{\mu}\right)$, and $\tau / \tau_{\mu} \ll 1:$

$$
\begin{gathered}
<\delta v^{2}>=\frac{\tau^{2}<\delta \omega_{e x}^{2}>}{\left(k_{a}+1\right)^{2}}\left[\theta_{f}\right]^{2} \\
<\delta \varphi^{2}>=\frac{\tau^{2}<\delta \omega_{e x}^{2}>}{k_{\varphi}{ }^{2}}\left[1+\theta_{f} k_{\mu} V_{0}^{2} \frac{2 \tau}{k_{a}+1}\left(1-\frac{\tau_{\mu}}{2 \tau} \tau^{2} \Omega_{\mu}^{2} \frac{k_{\varphi}+k_{a}+1}{k_{\varphi}\left(k_{a}+1\right)}\right)\right]
\end{gathered}
$$

In the absence of feedback phase shift, microphonics do not contribute to an amplitude error but cause a residual rms phase error $<\delta \varphi^{2}>^{1 / 2}=k_{\varphi}^{-1} \tau<\delta \omega_{e x}^{2}>^{1 / 2}$. Around $\theta_{f}=0$ the amplitude error is quadratic while the phase error is linear in $\theta_{f}$. This suggests that, if one is willing to accept a small amount of amplitude error, the phase error can be reduced by introducing a phase shift in the feedback signals $\left(\theta_{f} \neq 0\right)$. This is shown conceptually in Figure 3 .

This reduction in the residual phase error is a direct result of the reduction of the fluctuation in cavity frequency $\delta \omega_{c}=\delta \omega_{e x}+\delta \omega_{\mu}$. The amplitude variation $\delta v$ causes a modulation of the cavity frequency $\delta \omega_{\mu}$ through the radiation pressure that can counteract the variation $\delta \omega_{e x}$ due to microphonics. 

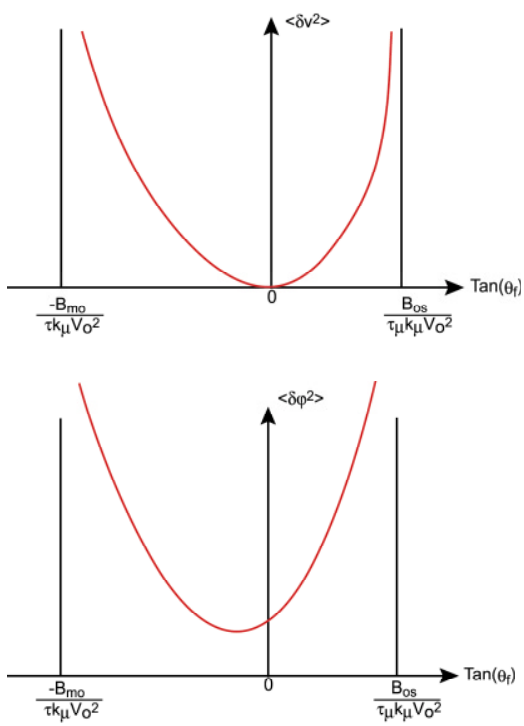

Figure 3: Conceptual representation of residual amplitude and phase errors as functions of the feedback phase. Both of them diverge as either the monotonic or oscillatory stability boundary is approached. See $[1,3]$.

\section{DAMPING BY FREQUENCY FEEDBACK}

The effectiveness of microphonics damping as described in the previous section depends on the amount of amplitude feedback, and is reduced as the feedback gain is increased. A more effective way to damp microphonics would be to intentionally modulate the amplitude reference by an amount dependent on the instantaneous frequency offset between the cavity and the master reference, and with the appropriate phase shift in order to act as a damping mechanism.

As shown in [3], in the absence of beam loading, and with no feedback phase shift $\left(\theta_{f}=0\right)$, the signal driving the resonator is of the form $V_{g}=V_{g o}\left[1+\delta v_{g}+i \delta t\right]$, where $\delta v_{g}=-F_{a} \delta v=-F_{a}(V-\mathrm{E}) / E$, and $\mathrm{E}$ is the amplitude reference. A modulation of the amplitude reference: $\mathrm{E}=\mathrm{E}_{0}(1+\delta e)$ introduces an additional term in the signal driving the resonator:

$$
\delta v_{g}(s)=-F_{a} \delta v(s)+\delta e(s) F_{a} .
$$

When the phase feedback gain $\left(k_{\varphi}\right)$ is sufficiently high, the "in quadrature" feedback signal $\delta t$ is directly proportional to the instantaneous phase error which, in turn, is proportional to the instantaneous difference between cavity and reference frequency. Thus $\delta t$ is an appropriate signal to provide a modulation of the amplitude reference: $\delta e(s)=-F_{\omega} \delta t(s)=\delta \varphi(s) F_{\varphi} F_{\omega}$, where $F_{\omega}$ is the frequency feedback transfer function.

In order to be effective as a damping mechanism, the frequency feedback needs to introduce a $\pi / 2$ phase shift between the frequency error and the amplitude modulation. For this reason, a good choice for $F_{\omega}$ is an integral-type feedback of the form:

$$
F_{\omega}=-k_{\omega} \frac{\Omega_{\mu}}{s} .
$$

The mean square frequency, phase, and amplitude errors can be calculated as in the previous section and are:

$$
\begin{aligned}
& <\delta \omega_{\mathrm{c}}^{2}>=<\delta \omega_{e x}^{2}>\frac{1}{1+k_{\omega} \tau \tau_{\mu} \Omega_{\mu} k_{\mu} V_{o}^{2}} \\
& <\delta \varphi^{2}>=\frac{\tau^{2}<\delta \omega_{e x}^{2}>}{k_{\varphi}^{2}} \frac{1}{1+k_{\omega} \tau \tau_{\mu} \Omega_{\mu} k_{\mu} V_{o}^{2}} \\
& <\delta v^{2}>=\tau^{2}<\delta \omega_{e x}^{2}>\frac{k_{\omega}^{2}}{1+k_{\omega} \tau \tau_{\mu} \Omega_{\mu} k_{\mu} V_{o}^{2}}
\end{aligned}
$$

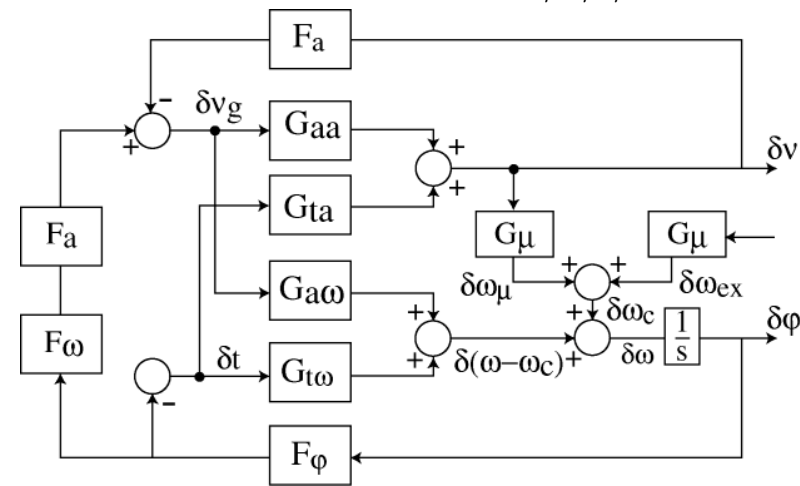

Figure 4: Block diagram with frequency feedback.

If $K$ is the reduction in frequency fluctuation: $\frac{<\delta \omega_{e x}^{2}>}{\left.<\delta \omega_{c}^{2}\right\rangle}=K^{2}$, the amplitude modulation needed to produce this reduction is: $\left\langle\delta v^{2}\right\rangle=\frac{\left\langle\delta \omega_{e x}^{2}\right\rangle}{4 Q_{\mu}^{2}\left(k_{\mu} V_{o}^{2}\right)^{2}} \frac{\left[K^{2}-1\right]^{2}}{K^{2}}$.

Assuming $K=2,<\delta \omega_{e x}^{2}>=(2 \pi \times 5)^{2},\left(k_{\mu} V_{o}^{2}\right)^{2}=(2 \pi \times 300)^{2}$, $\left(2 Q_{\mu}\right)^{2}=(2 \times 2 \pi \times 100 \times 0.25)^{2}$, gives $<\delta v^{2}>\simeq 0.610^{-8}$. An amplitude modulation of less than $10^{-4}$ could reduce the microphonics-induced frequency excursions, and the rf power needed to control them, by a factor of 2 .

\section{REFERENCES}

[1] J. R. Delayen, "Phase and Amplitude Stabilization of Superconducting Resonators", Ph.D. thesis, California Institute of Technology, 1978.

[2] J. R. Delayen, G. J. Dick, and J. E. Mercereau, “ A Microprocessor-Based Feedback System for Phase and Amplitude Stabilization of Superconducting Resonators, Proc. PAC 77, p. 1759.

[3] J. R. Delayen, "Phase and Amplitude Stabilization of Beam-Loaded Superconducting Resonators", Proc. Linac 92, p. 371.

[4] A. Facco, V. Zviagintsev, "Mechanical Stabilization of Superconducting Quarter-Wave Resonators", Proc. PAC 97, p. 3084. 